
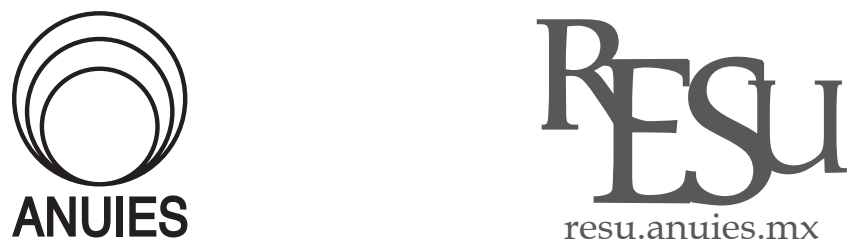

ARTíCULO

\title{
Presupuesto federal de educación superior en el primer año del gobierno de Andrés Manuel López Obrador: Negociaciones y retos
}

\author{
Higher Education Federal Budget in the First Year of the Andrés \\ Manuel López Obrador Government: Negotiations and Challenges
}

\author{
Javier Mendoza Rojas* \\ Financiamiento: El presente escrito se realizó con financiamiento del fondo SEP-CONACYT, convenio no. 131978, "Efectos \\ de las políticas gubernamentales en las Universidades Públicas Mexicanas (1989-2009): análisis comparativo de \\ su diversidad y complejidad" \\ * Investigador del Instituto de Investigaciones sobre la Universidad y la Educación de la UNAM. \\ correo electrónico: jmr0952@gmail.com
}

Recibido el 10 de junio de 2019/ Aceptado el 10 de septiembre de 2019

\section{Resumen}

En el artículo se analiza el proceso de negociación del presupuesto federal de educación superior contenido en el Presupuesto de Egresos de la Federación para el ejercicio fiscal 2019, correspondiente al primer año del gobierno de Andrés Manuel López Obrador en México. Se describe la forma como se corrigieron en la Cámara de Diputados las disminuciones presupuestales de las instituciones públicas de educación superior que consideraba el proyecto del Ejecutivo, y se presentan tres retos: la obligatoriedad de la educación superior establecida en el artículo tercero constitucional reformado; el costo de su gratuidad; la sostenibilidad financiera de las universidades públicas.

Palabras clave: Financiamiento de educación superior; Presupuesto de Egresos de la Federación; Política de educación superior; Universidades públicas 


\begin{abstract}
The paper analyses the negotiation process around Federal Expenditure Budget for Higher Education in 2019, corresponding to the first fiscal year of the new government lead by Andrés Manuel López Obrador in Mexico. It describes the rectifications done at the Mexican Congress from the original proposal sent by the Executive, which included significant cuts on the federal budget for public higher education institutions. It also presents three challenges: compulsory higher education as established in the recently reformed Article 3 of the Mexican Constitution; the cost of free higher education, and financial sustainability of public universities.
\end{abstract}

Keywords: Higher Education Financing; Federal Expenditure Budget; Higher Education Policy; Public Universities.

\title{
Introducción
}

$E_{p}^{1}$ Presupuesto de Egresos de la Federación (PEF) que la Cámara de Diputados aprueba para el primer año de una nueva administración federal constituye el instrumento central de las políticas públicas de las dependencias y entidades gubernamentales. De su diseño depende la viabilidad de los programas y líneas de acción presentados en la plataforma política de la fuerza política triunfante, así como de los compromisos establecidos con los electores durante la campaña política.

Andrés Manuel López Obrador, candidato presidencial de la coalición "Juntos Haremos Historia" - conformada por el Movimiento de Regeneración Nacional (Morena) como instituto político dominante, por el Partido del Trabajo (PT) y por el Partido Encuentro Social (PES) - triunfó en las elecciones del 1 de julio de 2018 con una amplia mayoría (53.2\%). En las dos cámaras del Congreso de la Unión, la de Diputados y la de Senadores, Morena obtuvo la mayoría absoluta, lo que modificó la correlación de fuerzas entre los partidos de las últimas dos décadas.

Por las fuerzas políticas contendientes en el proceso electoral de 2018 a nivel nacional y local, y por el indiscutible triunfo de los candidatos de Morena, el proceso de formulación del PEF adquirió características inéditas. El presupuesto estuvo determinado por las prioridades marcadas por la nueva administración, con la encomienda expresa del Presidente electo a las autoridades hacendarias entrantes de hacer ajustes a los programas presupuestarios existentes y canalizar recursos a los nuevos programas sociales y de inversión que constituirían los programas insignia de la nueva administración. A su vez, el presupuesto debería guiarse por los principios de austeridad, honestidad y combate a la corrupción. 
En el proceso de elaboración del presupuesto de educación superior intervinieron diversos actores: del lado gubernamental, los equipos salientes y entrantes de la Secretaría de Hacienda y Crédito Público (SHCP) y de la Secretaría de Educación Pública (SEP); del lado del Congreso, la Junta de Coordinación Política y las comisiones de Educación Pública y de Presupuesto y Cuenta Pública de la Cámara de Diputados; y por las universidades públicas la Asociación Nacional de Universidades e Instituciones de Educación Superior (ANUIES), los rectores y diversas agrupaciones sindicales.

Por tratarse del presupuesto correspondiente al primer año de una nueva administración federal, la fase de negociación del paquete económico en el Congreso transcurrió en tan solo nueve días, desde la presentación del proyecto por parte del Ejecutivo el 15 de diciembre, hasta la aprobación del PEF por el pleno de la Cámara de Diputados el 24 del mismo mes.

En este artículo se analiza el proceso de formulación negociación y aprobación del presupuesto de educación superior para el ejercicio fiscal 2019. El análisis se inscribe en el enfoque de investigación sobre los proceso de negociación política del Presupuesto de Egresos de la Federación, que se dan a partir del cambio en la composición política del Congreso en 1997 - cuando el Partido Revolucionario Institucional pierde la mayoría en la Cámara de Diputados-, y del triunfo del candidato del Partido Acción Nacional en el año 2000, inaugurando una nueva etapa en la interacción entre los poderes Ejecutivo y Legislativo y, al interior de éste, entre los grupos parlamentarios y las organizaciones de la sociedad civil. ${ }^{1}$

El artículo está dividido en dos partes. En la primera se describen los principales aspectos de su proceso de formulación y negociación: el cambio de régimen, los criterios para la evaluación de los programas presupuestarios; el proyecto del Ejecutivo; y las modificaciones realizadas en la Cámara de Diputados. En la segunda se revisan los programas prioritarios de educación superior de la nueva administración, y se presentan tres retos que tendrán que enfrentarse a partir de 2019: la obligatoriedad y universalización de la educación superior incorporada en el artículo tercero constitucional, de

\footnotetext{
${ }^{1}$ Ante el cambio de la correlación de fuerzas entre ambos poderes y al interior del legislativo, se ha conformado una línea de investigación en México sobre las relaciones emergentes entre los poderes ejecutivo y legislativo, el nuevo cabildeo en el Congreso y los procesos de negociación política del Presupuesto de Egresos de la Federación. Entre los estudios realizados se encuentran los realizados por organizaciones de la sociedad civil, como es el caso de Fundar (Dávila y Caballero, 2005); por empresas de consultoría, entre las que sobresale Integralia Consultores (Ugalde, 2014); por empresas de lobbying (Lerdo de Tejada, 2004), así como por investigadores universitarios (Casar, 2001; Heredia, 2004, y Galaviz, 2006). Para el caso del cabildeo presupuestal en educación superior de 2001 a 2007 se puede consultar el trabajo de Mendoza (2007).
} 
acuerdo con el decreto de reforma constitucional en materia educativa publicado el 15 de mayo de 2019; el costo de la gratuidad de este tipo educativo; y la sostenibilidad financiera de las universidades públicas a mediano plazo.

\section{De la transición a la aprobación del presupuesto de educación superior}

\section{El cambio de régimen}

El financiamiento de las instituciones públicas de educación superior constituyó uno de los temas de debate público durante el periodo de transición gubernamental entre los gobiernos de Peña Nieto y López Obrador a lo largo de los meses de julio a octubre de 2018, y durante la discusión y la aprobación del PEF para el Ejercicio Fiscal 2019.

A lo largo de los casi cuatro meses que duró la transición gubernamental se realizaron diversas reuniones entre las autoridades salientes y entrantes de la SEP y de la SHCP para revisar los escenarios para el presupuesto del sector educativo ${ }^{2}$, en un entorno político de cuestionamiento a la universidad pública en diversos espacios de la coalición política triunfante, pese a que las encuestas de opinión la colocaban entre las instituciones de mayor confianza de la población. Diversos legisladores propalaban una imagen de opacidad y corrupción en el funcionamiento de diversas universidades públicas; aún estaba presente en el ambiente político la investigación periodística conocida como la "estafa maestra" de 2013, que involucraba a funcionarios de la entonces Secretaría de Desarrollo Social (SEDESOL) y de la Secretaría de Desarrollo Agrario, Territorial y Urbano (SEDATU) y a diversas universidades públicas, en un esquema de desvío de recursos públicos. La crítica no distinguía entre actos de corrupción de funcionarios universitarios y la universidad en su conjunto, con lo cual se le juzgaba como institución opaca, ineficiente y privilegiada. Por su parte, las políticas de ciencia, tecnología e innovación

\footnotetext{
${ }^{2}$ En el período de transición, por parte del equipo entrante de Secretaría de Educación Pública, participaron quien ocuparía la titularidad de la SEP desde el 1 de diciembre de 2018, Esteban Moctezuma Barragán, y Luciano Concheiro Bórquez, quien sería nombrado Subsecretario de Educación Superior desde esa misma fecha. Por parte de la Secretaría de Hacienda y Crédito Público, el proyecto de PEF fue conducido por Carlos Urzúa Macías, quien ocuparía la titularidad del ramo, y por Gerardo Esquivel Hernández como encargado del área de la Subsecretaría de Egresos, quien sería designado Subgobernador del Banco de México en enero de 2019.
} 
impulsadas en la administración saliente del Consejo Nacional de Ciencia y Tecnología (CONACYT) fueron calificadas por el equipo entrante en ese organismo de neoliberales y favorecedoras de las grandes empresas. Cabe señalar que, por las formas en que se realizó el proceso de negociación del presupuesto para educación superior, el equipo entrante de la SEP no compartió la postura de descalificación de la universidad pública.

Uno de los ejes de la campaña de López Obrador había sido la austeridad gubernamental y el combate a la corrupción. Durante los meses de transición, en su nuevo recorrido por todos los estados, fortaleció el discurso anticorrupción y, una vez en el gobierno, las conferencias matutinas fueron un espacio privilegiado de comunicación para dar a conocer actos de malversación de fondos en diversos sectores del gobierno federal.

Desde el inicio de la transición, el equipo entrante de la sHCP señaló que se reorientaría el PEF a fin de destinar recursos a los nuevos programas sociales anunciados en la campaña: aumento a la pensión a las personas adultas mayores, pensión a las personas con discapacidad, beca universal a estudiantes de educación media superior; apoyos a los jóvenes desempleados para su inserción en empresas, apertura de cien universidades en zonas marginadas, medicamentos gratuitos a toda la población, etc. Junto a los programas sociales, se impulsarían los proyectos de inversión prioritarios en el nuevo gobierno, entre ellos el tren maya y la nueva refinería en Tabasco. Estimaciones realizadas por el equipo hacendario situaban los requerimientos para los programas emblemáticos del nuevo gobierno en el orden de los 500 mil millones de pesos.

Para disponer de estos recursos se establecieron como principios la austeridad, la honestidad y el combate a la corrupción. Ello llevó a plantear medidas de ahorro como la reducción de servicios personales, la revisión de los fondos y fideicomisos (principalmente del Ramo 23, Provisiones Salariales y Económicas, conocido coloquialmente como el "ramo de los moches") y la revisión del desempeño de los programas presupuestarios del ámbito social. Como parte de la política de austeridad se anunció que ningún funcionario público podría ganar más que el Presidente, en los términos que se establecieran en la legislación sobre las percepciones de los servidores públicos, además de que se eliminarían los gastos para el seguro de separación individualizado y la contratación de seguros de gastos médicos mayores de los funcionarios. La ley fijó el salario del presidente, el cual sería el tope de ingresos para todos los servidores públicos. El recorte a la burocracia de las dependencias sería una medida adicional que afectaría principalmente al personal contratado por honorarios, con impacto en algunos organismos académicos del país. 
Junto con la política de austeridad y la reorientación del gasto, se anunció que en el paquete económico para 2019 no se recurriría a mayor deuda pública ni al incremento de impuestos, de ahí que la encomienda presidencial al equipo hacendario implicara realizar ajustes a la baja en diversos programas presupuestarios vigentes a fin de fondear los nuevos programas sociales y de inversión. Lo prioritario era contar con recursos para cumplir con los compromisos de campaña del presidente.

La negociación del presupuesto para 2019 se verificó no sólo en el contexto de un reordenamiento de los partidos políticos - en el cual los partidos de la coalición política triunfante obtuvieron mayoría absoluta en el Congreso (63\% en la Cámara de Diputados y 55\% en el Senado) - , sino en el marco de un proyecto de cambio régimen, que fue denominada la "cuarta transformación" (de acuerdo con la narrativa presidencial, las primeras tres transformaciones habrían sido la Independencia, la Reforma y la Revolución).

El nuevo grupo hegemónico tenía las condiciones políticas en el Ejecutivo y en el Congreso para desmontar el modelo de desarrollo impulsado en las tres últimas décadas tanto en su dimensión económica (concentración de la riqueza y exclusión de las mayorías en el desarrollo del país), como educativa (homogeneización cultural y evaluación punitiva de los maestros a partir de la denominada "mal llamada" reforma educativa del gobierno de Enrique Peña Nieto). El proyecto de la cuarta transformación, la priorización de los programas sociales, y los principios de austeridad, honestidad y combate a la corrupción, determinarían la elaboración del PEF.

Cabe señalar que en los tres años que precedieron el arranque del nuevo gobierno, la SHCP había realizado diversos ajustes presupuestales. Como ha sido documentado en otro trabajo (Mendoza, 2017), a mediados del sexenio de Peña Nieto llegó a su fin una etapa de las finanzas públicas sostenidas. La caída de los ingresos petroleros de 2015 y la volatilidad de la economía mundial llevaron a las autoridades hacendarias a practicar tres recortes al presupuesto, uno en 2015 y dos en 2016, ${ }^{3}$ que afectaron a diversos programas destinados a las instituciones públicas de educación superior. ${ }^{4}$

\footnotetext{
${ }^{3}$ A la crisis petrolera de 2015 se agregaron dos situaciones: la salida del Reino Unido de la Unión Europea (Brexit) en el referéndum realizado a mediados de 2016, y el triunfo de Donald Trump en las elecciones presidenciales de Estados Unidos en el mes de noviembre de ese año, que repercutió en la devaluación del peso frente al dólar y en la mayor incertidumbre sobre el desempeño de la economía mexicana. La volatilidad de la paridad del peso se vio reforzada por las declaraciones de Trump en el sentido de renegociar el Tratado de Libre Comercio y tomar otras medidas de carácter proteccionista que afectarían a la economía mexicana.

${ }^{4}$ Desde el establecimiento de las políticas impulsadas por la SEP en los inicios de los años
} 
Con estas medidas, las universidades y demás instituciones de educación superior enfrentaban retos para la sostenibilidad de sus finanzas institucionales y el desempeño de sus funciones académicas. Como veremos más adelante, los recortes a los fondos extraordinarios para educación superior continuarían en 2019.

\section{Evaluación de los Programas Presupuestarios}

A finales de septiembre de 2018, como un insumo para la formulación del proyecto de PEF para el ejercicio fiscal 2019, el equipo de transición del área de la Subsecretaría de Egresos de la SHCP dio a conocer al correspondiente equipo de transición de la SEP los resultados del análisis sobre la prescindibilidad de programas presupuestarios, con base en una metodología cuantitativa que denominaron "Índice de Prescindibilidad de Programas Presupuestarios (I3P)" y una revisión de tipo cualitativo. ${ }^{5}$ El propósito del análisis que se realizó a los 156 programas sociales de la administración pública federal, fue “[...] conocer el estatus actual de la oferta institucional de la política social en términos de diseño, implementación y resultados, y así poder identificar potenciales áreas de mejora que permita a las dependencias y entidades contar con elementos para llevar a cabo el rediseño programático inherente a un cambio de administración pública" (SHCP, 2018a: 24). De acuerdo con la información presentada en la exposición de motivos del proyecto de PEF, se consideró que, de todos los programas sociales vigentes en 2018 , el $48 \%$ eran altamente prescindibles, el $31 \%$ tenían una prescindibilidad media, el 14\% una imprescindibilidad baja y solo el 7\% de ellos se consideraban imprescindibles (SHCP, 2018a: 26).

noventa del siglo pasado (políticas de modernización de la educación), reforzadas a partir del año 2000, los fondos extraordinarios habían constituido el instrumento clave para el impulso de proyectos de cambio institucional. De 2007 a 2015 los diversos fondos, que llegaron a sumar más de una decena en 2010, venían observando una tendencia de crecimiento presupuestal constante, lo que se revierte a partir de 2015. Si se compara el presupuesto ejercido anualmente en estos fondos de 2015 a 2018 con el presupuesto aprobado para el primero de esos años, los recortes acumulados sumaron más de 22 mil millones de pesos. Estos ajustes impactaron negativamente en el desarrollo de diversos programas de expansión de matrícula, fortalecimiento de la calidad y atención a problemas estructurales de las universidades públicas.

${ }^{5}$ Para el análisis se consideraron, entre otros elementos, las evaluaciones de los programas presupuestarios realizadas por el Consejo Nacional de Evaluación de la Política Social, las consideraciones que hizo este organismo para el proceso presupuestario 2019 (CONEVAL, 2018), así como los resultados de auditorías realizadas por la Auditoría Superior de la Federación. 
En el caso de la SEP, todos sus programas presupuestarios fueron evaluados con esta metodología, con la cual el equipo de transición hacendario formuló propuestas al equipo entrante para la continuación, la modificación o la eliminación de programas presupuestarios. Se consideró que el 70\% de los programas eran altamente prescindibles, lo que apuntaba a una reordenación profunda del gasto con impactos en diversos ámbitos del quehacer educativo. Varios programas educativos fueron considerados prescindibles por no contar con las características de un programa social, es decir, aquel que contribuye de manera clara al acceso de los derechos sociales y a la reducción de alguna carencia social, y cuyos beneficiarios directos son las personas de mayor pobreza o vulnerabilidad. Bajo la lógica de que aquellos programas que no entran en esta definición eran prescindibles, o bien requerían de una cirugía mayor, se ponía en cuestionamiento la continuidad de las acciones impulsadas por la SEP para el fortalecimiento de las IES, tanto en su operación ordinaria como para la realización de proyectos de fortalecimiento académico e institucional. Las sugerencias del equipo hacendario implicaba frenar proyectos para el mejoramiento de la calidad, además de no contar con recursos para la expansión de la oferta educativa y para la atención a los problemas estructurales de las universidades, particularmente en el tema relacionado con los sistemas de pensiones y jubilaciones. ${ }^{6}$

La insuficiencia de recursos para la operación de las universidades a través del Pp U006 (Subsidios para organismos descentralizados estatales) generaría, además, ingobernabilidad institucional con diversos impactos políticos y sociales en las entidades federativas: incumplimiento de las obligaciones contractuales, paros, huelgas y movilización de profesores y trabajadores administrativos. En esos días nueve universidades públicas estatales estaban imposibilitadas de cerrar el año con el pago de salarios y aguinaldo para el mes diciembre, las cuales, desde meses atrás, venían negociando con la SEP y con la SHCP ampliaciones extraordinarias. En una de ellas (Universidad Autónoma de Morelos) el sindicato académico había estallado una huelga el 20 de septiembre ante la falta de pago de salarios.

\footnotetext{
${ }^{6}$ La Oficialía Mayor de la SEP, al igual que todas las secretarías de Estado, se transformó en la Unidad de Administración y Finanzas (UAF), pasando a ser la correa de transmisión directa de las decisiones de la SHCP a las autoridades educativas, con nuevas atribuciones en materia presupuestaria y de control del gasto. La UAF de la SHCP centralizó las compras de toda la administración pública federal, con un poder de decisión que afectaría la operación de todas las dependencias de la administración pública federal a lo largo del primer semestre de 2019 (desabasto de medicamentos, retraso en adquisiciones y en licitaciones de libros de texto, entre otros).
} 
Resultado del proceso de negociación descrito, las autoridades salientes de la SEP dieron a conocer a los titulares de las instituciones federales de educación superior, mediante oficio, los techos presupuestales que la SHCP consideraba en el anteproyecto para la integración en el PEF 2019, los cuales consideraban recortes. Por otra parte el diario Reforma, en su edición del 19 de noviembre, dio a conocer que la SHCP planteaba disminuir la tercera parte del presupuesto ordinario a las universidades públicas estatales, ante lo cual Gerardo Esquivel, quien había sido invitado por el Presidente electo a ocupar la Subsecretaría de Egresos de la SHCP, señaló vía Twitter: “Es falso que el presupuesto destinado a las universidades públicas en 2019 se reducirá en $32 \%$. El proyecto de presupuesto que propondrá la nueva administración será dado a conocer en tiempo y forma". Ese mismo día Carlos Urzúa, futuro Secretario de Hacienda, reconoció que hubo un error de cálculo de los sueldos y salarios: "[...] se les dieron los nuevos tabuladores y fue un errorcito. Ya se compuso" (Rodríguez, 2018).

En este clima de comunicaciones contradictorias y de incertidumbre sobre el presupuesto, los rectores de las universidades mantuvieron una postura de cautela y acordaron en la Sesión Ordinaria de la Asamblea General de la ANUIES, reunida el 20 de noviembre en Mazatlán, Sinaloa, esperar a la presentación oficial del proyecto de presupuesto del gobierno que iniciaría funciones el 1 de diciembre, recordando que el Presidente electo se había comprometido con la ANUIES a no reducir el presupuesto a las universidades.

\section{Proyecto de Presupuesto de Egresos de la Federación}

En cumplimiento a los plazos establecidos en la Ley de Presupuesto y Responsabilidad Hacendaria, el 15 de diciembre el Congreso de la Unión recibió del Ejecutivo la Iniciativa de Ley de Ingresos y el Proyecto de Presupuesto de Egresos de la Federación (SHCP, 2018b).

El proyecto de presupuesto para educación superior modificó algunos de los recortes del anteproyecto de presupuesto. La principal rectificación fue el subsidio ordinario de las universidades e instituciones estatales (Pp U006 Subsidios para organismos descentralizados estatales), al presentar el mismo monto del presupuesto aprobado en 2018, pero sin considerar la inflación. Para las IES federales, en conjunto, el presupuesto disminuía en 1,714 mdp respecto al presupuesto aprobado para 2018; ello equivalía a una reducción real del 5.7\%: a la UNAM se le disminuían 1,023 mdp (-6.3\%); a la UAM 290 mdp $(-7.7 \%)$ y al IPN 168 mdp (4.7\%). Los fondos extraordinarios también tenían reducciones importantes. Solamente se mantenían, con pocas variaciones, 
el Programa de Carrera Docente para las universidades públicas estatales y el programa de Apoyo a Centros y Organizaciones de Educación. El Programa de Desarrollo Profesional Docente (PRODEP) disminuía en 410 mdp (-63\%), el Programa de Fortalecimiento de la Calidad Educativa en 746 mdp (-42\%) y el Programa para la Inclusión y la Equidad Educativa en 15 mdp (-37\%). No contaron con recursos los programas de Expansión de la Educación Media Superior y Superior y de Apoyos para la Atención a Problemas Estructurales de las UPES.

El presupuesto del Programa Nacional de Becas para la Subsecretaría de Educación Superior y la Dirección General de Educación Superior para Profesionales de la Educación tenían una disminución de 2,692 mdp (-53\%), y se establecían dos nuevos programas: Universidades para el Bienestar Benito Juárez García con 1,000 mdp y Jóvenes Construyendo el Futuro con 4,320 mdp. La disminución presupuestal de las IES federales y de los programas existentes, por un monto superior a los 6 mil mdp, se trasladaba a los dos nuevos programas, con lo cual se daba cumplimiento a la encomienda presidencial de dar prioridad a los nuevos programas sociales.

En el proyecto de PEF los ajustes no se hacían solamente para las IES públicas; otros programas e instituciones educativas presentaban recortes, al igual que programas de organismos autónomos, secretarías, dependencias. Entre los primeros, el Instituto Nacional Electoral (INE) disminuía en 35\% y el Instituto Nacional de Evaluación de la Educación (INEE) en 22\%; para el conacyt se planteaba una reducción del 13\%, y para la Secretaría de Cultura de $7.6 \%$. Otras dos secretarías afectadas de manera importante eran las secretarías del Medio Ambiente (SEMARNAT) y de Agricultura y Desarrollo Rural (SADER), las cuales disminuían su presupuesto en alrededor de la tercera parte. A diferencia de ello, otras dependencias federales tenían incrementos; entre las más beneficiadas estaban la Secretaría del Bienestar (antes Secretaría de Desarrollo Social) con $41 \%$ de aumento y la Secretaría de Turismo con 16\%. La Secretaría del Trabajo y Previsión Social tenía el mayor incremento por la creación del Programa Jóvenes Construyendo el Futuro en su modalidad de incorporación de aprendices a las empresas, con un presupuesto de 40 mil mdp.

Las reacciones al proyecto de presupuesto para las instituciones públicas de educación superior no se hicieron esperar. El Consejo Nacional de la ANUIES señaló en desplegado público que López Obrador, en reunión con la Asociación el 15 de agosto, "[...] se comprometió ante los rectores y directores de las instituciones de educación superior más importantes del país a no reducir el presupuesto del presente año destinado a las universidades públicas y a los tecnológicos, más la compensación por la inflación" (ANUIEs, 2018b), y solicitó a la Cámara de Diputados realizar los ajustes necesarios para 
otorgar los recursos que requiere la educación superior. En el mismo sentido se pronunciaron la UNAM, la UAM y diversas universidades públicas estatales.? El compromiso de López Obrador de no reducir el presupuesto en términos reales constituyó el argumento central que utilizaron los rectores en la defensa del presupuesto. Además de los anteriores pronunciamientos, diversos sindicatos universitarios se manifestaron en contra del proyecto, y trabajadores y estudiantes de la UNAM, la UAM y el IPN habían convocado a manifestarse en las calles, además de que nueve universidades públicas estatales estaban imposibilitadas a cubrir el pago de salarios y prestaciones para cerrar el año.

En este clima de inconformidad, el Secretario de Hacienda, en la comparecencia en la Cámara de Diputados para el análisis del proyecto de PEF, defendió la reducción al presupuesto a las universidades, señalando que “[...] anteriormente el gasto público no se ejercía de forma correcta en distintas dependencias, poderes y organismos autónomos, lo cual en esta administración no sucederá" (El Heraldo de México, 2018). El cuestionamiento a las universidades por la forma como se ejercen los recursos estaba en el trasfondo de la propuesta presentada por el equipo hacendario del nuevo gobierno, el cual se sustentaba en el discurso presidencial de combate a la corrupción.

Al ser cuestionado López Obrador en una de sus conferencias de prensa matutinas sobre el recorte presupuestal a UNAM, dijo desconocer la existencia de recortes a las universidades; señaló que el presupuesto se incrementaba con los recursos que se destinarían a los programas Jóvenes Construyendo el Futuro y Universidades para el Bienestar Benito Juárez García, y aclaró que se haría más con menos porque "se terminarían con lujos" y habría "austeridad republicana" (Nación 321, 2018). De acuerdo con estos pronunciamientos, el titular del Ejecutivo no habría sido informado sobre los recortes a las universidades; su apreciación era que el presupuesto de educación superior se incrementaba al asignar más de 5 mil mdp a los dos nuevos programas.

\footnotetext{
${ }^{7}$ La UNAM señaló: “La sorpresiva e inusitada propuesta de reducir el presupuesto de la UNAM en más de seis por ciento no corresponde a la trascendente e importante labor de la institución de enseñanza superior más grande de América Latina ni al esfuerzo cotidiano que realiza para atender a una comunidad de más de 350 mil alumnos" (El Financiero, 2018). La unAm consideró "inaceptable" la reducción propuesta de $7.7 \%$ y alertó que una decisión de este tipo "pone en riesgo el desarrollo de sus labores académicas, así como el crecimiento programado de su matrícula y la necesaria ampliación de su infraestructura" (Román, José Antonio, 2018). En un desplegado firmado por 16 rectores de universidades públicas estatales, se afirmó que el proyecto de PEF enviado por la SHCP no cumple la garantía externada por el Presidente de que no habría reducción presupuestal, y solicitó rectificar para evitar cualquier afectación a los procesos educativos de las universidades (Reporte Índigo, 2018).
} 
Sin embargo, al día siguiente de esta conferencia, al encabezar la reapertura de la escuela normal rural El Mexe - que formaría parte del nuevo sistema de universidades para el Bienestar - reconoció que se cometió un error en la presentación del presupuesto de las universidades públicas y que sería corregido. Lo dijo en los siguientes términos:

Se va a reparar ese error y se va a entregar a las universidades lo que les corresponde, lo que acordamos, lo que dije en una reunión de la ANUIES. Dije que si no aumentaba el presupuesto, iba a ser el mismo del 2018 más inflación. Ese es mi compromiso y lo voy a cumplir. Lo dije pero no se registró en el presupuesto y esto ha creado inconformidades, y en mi gobierno, cuando se cometa un error vamos a rectificar. ¿Qué les pido nada más a los rectores?, que nos ayuden a que haya austeridad en el manejo del presupuesto de las universidades. Voy a ser respetuoso de la autonomía de las universidades, pero quiero que haya honradez en el manejo del presupuesto que se dirige a las universidades públicas. Se acaba la corrupción. (Hernández, 2018).

En esos días los rectores, agrupados en la ANUIES, sostuvieron diversas reuniones con diputados de la Junta de Coordinación Política y de las comisiones de Educación Pública y de Presupuesto y Cuenta Pública, en las que presentaron solicitudes de modificación del proyecto del Ejecutivo para dar cumplimiento al compromiso de mantener en términos reales el presupuesto ordinario a las universidades, además de no afectar los fondos extraordinarios.

El compromiso presidencial de rectificación del presupuesto para las universidades fue posible por la bolsa adicional de recursos que consideró la Ley de Ingresos aprobada por ambas cámaras los días 19 y 20 de noviembre. Con las modificaciones realizadas a la iniciativa de Ley presentada por el Ejecutivo, se estimaron ingresos adicionales cercanos a los 24 mil mdp, monto disponible para las ampliaciones al PEF.

\section{Modificaciones en la Cámara de Diputados}

Una vez aprobada la Ley de Ingresos, en un período de tan solo cinco días, los diputados se dieron a la tarea de hacer los ajustes al Proyecto de PEF. Las comisiones ordinarias recibieron alrededor de 60 mil peticiones de recursos federales por un monto cercano a un billón de pesos, y solicitaron a la Comisión de Presupuesto y Cuenta Pública ampliaciones al Proyecto de PEF por un monto superior a 600 mil mdp. (la Comisión de Educación Pública recibió 
más de 5 mil peticiones por $31 \mathrm{mil} \mathrm{mdp).} \mathrm{Sin} \mathrm{embargo,} \mathrm{solamente} \mathrm{se} \mathrm{disponía}$ de una bolsa adicional de 24 mil mdp. (Comisión de Presupuesto, 2018:7-9).

La Comisión de Presupuesto y Cuenta Pública de la Cámara de Diputados condujo el proceso de negociación para ajustar el Proyecto de PEF, en coordinación con las autoridades de la sHCP y con la Junta de Coordinación Política. El 23 de diciembre la comisión aprobó el dictamen con 33 votos a favor y 14 en contra, y el día 24 se aprobó en el pleno, con el voto mayoritario (312) de los diputados de Morena, Partido Encuentro Social, Partido del Trabajo, Partido Verde y la mitad de los diputados del Partido de la Revolución Democrática; en contra votaron los diputados del Partido Revolucionario Institucional, del Partido Acción Nacional y de Movimiento Ciudadano.

En el dictamen se hicieron distintos ajustes al proyecto presupuestal, con reducciones en distintos ramos por 16,759 mdp y ampliaciones por 40,525 mdp, con lo cual el incremento neto fue de 23,768 mdp, cantidad ampliada en la Ley de Ingresos. Las secretarías y los ramos con mayores ampliaciones fueron SEP (7,860 mdp); Participaciones a Entidades Federativas y Municipios (5,321 mdp); SADER (8,092 mdp) y SEMARNAT (4,500 mdp). Las reducciones se hicieron principalmente al Poder Judicial (-1,700 mdp) y al Instituto Nacional Electoral (-950 mdp), órgano autónomo al que se le redujo aún más su presupuesto, por lo que presentó un amparo.

La mayor parte de las ampliaciones a la SEP se destinaron a educación superior, con lo cual se dio cumplimiento al compromiso que López Obrador había hecho con la ANUIEs. ${ }^{8}$ De los 6,960.2 mdp de ampliaciones a educación superior, a las instituciones federales sectorizadas en la SEP (exceptuando a la Universidad Abierta y a Distancia de México) se asignaron 4,288.2 mdp, y para la Universidad Autónoma Chapingo, sectorizada en SAGARPA, se acordó una ampliación por 91.8 mdp. Por su parte, el subsidio ordinario de los organismos descentralizados estatales (universidades públicas estatales, con apoyo solidario, interculturales, tecnológicas y politécnicas, e institutos tecnológicos descentralizados), se amplió en 2,672 mdp. La solicitud de la ANUIES de no afectar los fondos extraordinarios no prosperó.

De este modo, el presupuesto ordinario para las IES federales y estatales mantuvo el valor real del presupuesto aprobado para 2018, sin considerar los incrementos salariales otorgados en ese año, por lo que se verían en la necesi-

\footnotetext{
${ }^{8}$ Para los ajustes se aplicó la variación del deflactor del PIB de 3.85\% establecida en los Criterios Generales de Política Económica para 2019.
} 
dad de contar con recursos adicionales para cubrir la política salarial de 2019, ${ }^{9}$ que sería uno de los motivos de los estallamientos de huelga en diversas universidades en el primer trimestre del año. ${ }^{10}$

El presupuesto en las subfunciones de educación superior y posgrado de los distintos ramos presupuestales ascendió a 135,199 mdp, y observó una variación real de $2.1 \%$. El incremento fue resultado de la creación de los dos nuevos programas presupuestarios (Jóvenes Construyendo el Futuro y Universidades para el Bienestar Benito Juárez García).

Los fondos extraordinarios existentes disminuyeron en conjunto poco más del 50\%; el más afectado fue el Pp U081 Apoyos para Reformas Estructurales de las upe, ya que por primera vez desde su creación no contaba con presupuesto. ${ }^{11}$ Adicionalmente, por segundo año consecutivo, no se consideraban recursos para educación superior en el Pp U079 Expansión de la Educación Media Superior y Superior, en tanto dos de los fondos para la mejora de la calidad tuvieron fuertes disminuciones: el Programa para el Desarrollo Profesional Docente disminuyó 64\% y el programa de Fortalecimiento de la Calidad Educativa lo hizo en $42 \%$, acentuando la curva de disminución presupuestal iniciada en 2015.

En los siguientes cuadros se presentan las variaciones del presupuesto en las subfunciones de educación superior y posgrado, en las IES federales y en el subsidio ordinario y extraordinario de las IEs estatales.

\footnotetext{
${ }^{9}$ Con la política de SEP y de SHCP del tope salarial de $3.35 \%$, que fue el que obtuvo el STUNAM en la revisión salarial con la UNAM en noviembre de 2018

${ }^{10}$ El Sindicato Independiente de la Universidad Autónoma Metropolitana estalló la huelga en febrero de 2019 con la demanda de $20 \%$ de incremento salarial y la revisión al contrato colectivo de trabajo; la postura de las autoridades de no romper el 3.35\% de incremento directo al asalario hizo que se prolongara el movimiento durante 92 días, rompiendo el récord de duración de una huelga laboral en dicha institución. Las universidades autónomas Chapingo y Agraria Antonio Narro, además de la Universidad de Sonora, también estallaron huelgas por demandas salariales superiores al tope salarial establecido por la SHCP.

${ }^{11}$ Los proyectos de PEF de 2017 y 2018 no consideraron recursos para el Pp U081; en ambos años la Cámara de Diputados aprobó ampliaciones por 600 y 700 mdp. Para 2019 el proyecto de presupuesto tampoco consideró recursos, si bien se tenía la expectativa de que nuevamente los legisladores corrigieran el proyecto del Ejecutivo, cosa que no ocurrió.
} 


\section{Cuadro 1 \\ Presupuesto para las Subfunciones de Educación Superior y de Posgrado (Millones de pesos)}

\begin{tabular}{|c|c|c|c|c|c|c|}
\hline \multirow{2}{*}{ Gasto / Ramo } & \multirow{2}{*}{\multicolumn{2}{|c|}{2018 a }} & \multirow{2}{*}{2019 a } & \multicolumn{3}{|c|}{ Variación 2018 a - 2019 a } \\
\hline & & & & \multicolumn{2}{|l|}{ Nominal } & $\%$ real $^{1 /}$ \\
\hline Ramo 11 (SEP) & $117,828.3$ & \multicolumn{2}{|c|}{$124,893.7$} & $7,065.4$ & \multicolumn{2}{|c|}{$2.1 \%$} \\
\hline Ramo 07 (SEDENA) & $1,316.1$ & \multicolumn{2}{|c|}{$1,420.3$} & 104.2 & \multicolumn{2}{|c|}{$3.9 \%$} \\
\hline Ramo 08 (SAGARPA) & $3,287.8$ & \multicolumn{2}{|c|}{$3,308.3$} & 20.5 & \multicolumn{2}{|c|}{$-3.1 \%$} \\
\hline Ramo $33^{2 /}$ & $3,959.7$ & \multicolumn{2}{|c|}{$4,521.6$} & 561.9 & \multicolumn{2}{|c|}{$9.9 \%$} \\
\hline Ramo $25^{3 /}$ & $1,084.9$ & \multicolumn{2}{|c|}{$1,055.4$} & -29.5 & \multicolumn{2}{|c|}{$-6.3 \%$} \\
\hline TOTAL & $127,476.8$ & \multicolumn{2}{|c|}{$135,199.3$} & $7,722.5$ & \multicolumn{2}{|c|}{$2.1 \%$} \\
\hline \multicolumn{7}{|c|}{$\begin{array}{l}\text { Económica para la Iniciativa de Ley de Ingresos y el Proyecto de Presupuesto c } \\
\text { 2019. } \\
\text { 2/ Aportaciones Federales para Entidades Federativas y Municipios. } \\
\text { 3/ Previsiones y Aportaciones para los Sistemas de Educación Básica, Normal, }\end{array}$} \\
\hline \multicolumn{7}{|c|}{ Fuente: Presupuesto de Egresos de la Federación para los Ejercicios Fiscales 2018 y 2019.} \\
\hline \multicolumn{7}{|c|}{$\begin{array}{c}\text { Cuadro } 2 \\
\text { Variaciones del Presupuesto de Instituciones Federales de Educación Superior, 2018-2019 } \\
\text { (Millones de pesos) }\end{array}$} \\
\hline \multirow{2}{*}{ Institución } & \multirow{2}{*}{2018 a } & \multirow{2}{*}{\multicolumn{2}{|c|}{$\begin{array}{l}\text { Ampliaciones } \\
\text { Cámara de } \\
\text { Diputados }\end{array}$}} & \multirow{2}{*}{2019 a } & \multicolumn{2}{|c|}{$\begin{array}{c}\text { Variación } 2018 \text { a - } \\
2019 \text { a }\end{array}$} \\
\hline & & & & & Absoluta & $\%$ real $^{1 /}$ \\
\hline $\begin{array}{l}\text { Universidad } \\
\text { Nacional } \\
\text { Autónoma de } \\
\text { México }\end{array}$ & $38,300.4$ & $37,277.4$ & $2,501.3$ & $39,778.7$ & $1,478.3$ & $0.0 \%$ \\
\hline $\begin{array}{l}\text { Universidad } \\
\text { Autónoma } \\
\text { Metropolitana }\end{array}$ & $7,028.2$ & $6,738.5$ & 561.0 & $7,299.5$ & 271.3 & $0.0 \%$ \\
\hline $\begin{array}{l}\text { Instituto } \\
\text { Politécnico } \\
\text { Nacional }\end{array}$ & $16,348.9$ & $16,181.1$ & 798.8 & $16,979.9$ & 631.0 & $0.0 \%$ \\
\hline $\begin{array}{l}\text { Tecnológico } \\
\text { Nacional de } \\
\text { México } 2 /\end{array}$ & $15,981.7$ & $16,510.0$ & 137.9 & $16,647.6$ & 665.9 & $0.3 \%$ \\
\hline $\begin{array}{l}\text { Universidad } \\
\text { Autónoma Agraria } \\
\text { Antonio Narro }\end{array}$ & 971.6 & 920.6 & 88.5 & $1,009.1$ & 37.4 & $0.0 \%$ \\
\hline $\begin{array}{l}\text { El Colegio de } \\
\text { México }\end{array}$ & 582.4 & 554.3 & 50.6 & 604.9 & 22.5 & $0.0 \%$ \\
\hline $\begin{array}{l}\text { Centro de } \\
\text { Investigaciones } \\
\text { y Estudios } \\
\text { Avanzados del IPN }\end{array}$ & $2,260.3$ & $2,203.2$ & 144.3 & $2,347.5$ & 87.2 & $0.0 \%$ \\
\hline
\end{tabular}

Continúa... 


\begin{tabular}{|c|c|c|c|c|c|c|}
\hline \multirow{2}{*}{ Institución } & \multirow{2}{*}{2018 a } & \multirow{2}{*}{$2019 p$} & \multirow{2}{*}{$\begin{array}{l}\text { Ampliaciones } \\
\text { Cámara de } \\
\text { Diputados }\end{array}$} & \multirow{2}{*}{2019 a } & \multicolumn{2}{|c|}{$\begin{array}{c}\text { Variación } 2018 \text { a - } \\
2019 \text { a }\end{array}$} \\
\hline & & & & & Absoluta & $\%$ real $^{1 /}$ \\
\hline $\begin{array}{l}\text { Universidad } \\
\text { Abierta y a } \\
\text { Distancia de } \\
\text { México }\end{array}$ & 202.4 & 193.8 & 0.0 & 193.8 & -8.5 & $-7.8 \%$ \\
\hline $\begin{array}{l}\text { Comisión de } \\
\text { Operación y } \\
\text { Fomento de } \\
\text { Actividades } \\
\text { Académicas del IPN }\end{array}$ & 233.8 & 203.2 & 39.6 & 242.8 & 9.0 & $0.0 \%$ \\
\hline $\begin{array}{l}\text { Patronato de Obras } \\
\text { e Instalaciones } \\
\text { del IPN }\end{array}$ & 41.9 & 35.0 & 8.4 & 43.4 & 1.5 & $-0.2 \%$ \\
\hline $\begin{array}{l}\text { Centro de } \\
\text { Enseñanza Técnica } \\
\text { Industrial }\end{array}$ & 332.6 & 306.2 & 39.1 & 345.3 & 12.7 & $0.0 \%$ \\
\hline $\begin{array}{l}\text { Universidad del } \\
\text { Ejército y la Fuerza } \\
\text { Aérea }\end{array}$ & $1,829.6$ & $1,992.7$ & 0.0 & $1,992.7$ & 163.2 & $4.9 \%$ \\
\hline $\begin{array}{l}\text { Universidad } \\
\text { Autónoma } \\
\text { Chapingo }\end{array}$ & $2,717.2$ & $2,730.3$ & 91.8 & $2,822.1$ & 104.9 & $0.0 \%$ \\
\hline $\begin{array}{l}\text { Colegio de } \\
\text { Posgraduados }\end{array}$ & $1,355.8$ & $1,361.1$ & 0.0 & $1,361.1$ & 5.3 & $-3.3 \%$ \\
\hline $\begin{array}{l}\text { Colegio Superior } \\
\text { Agropecuario } \\
\text { del Estado de } \\
\text { Guerrero }\end{array}$ & 110.1 & 110.2 & 0.0 & 110.2 & 0.1 & $-3.6 \%$ \\
\hline TOTAL & $89,123.2$ & $88,119.3$ & $4,517.9$ & $92,636.9$ & $3,513.7$ & $0.1 \%$ \\
\hline
\end{tabular}

1/ Crecimiento del Índice de precios implícitos del pib para 2019 de 3.9\%, de acuerdo con los Criterios Generales de Política Económica para la Iniciativa de Ley de Ingresos y el Proyecto de Presupuesto de Egresos de la Federación para el Ejercicio Fiscal 2019.

2/ No incluye el Pp U006 para los Institutos Tecnológicos Descentralizados.

a - aprobado.

$\mathrm{p}$ - proyecto.

Fuente: Elaboración propia con datos del Proyecto de Presupuesto de Egresos de la Federación 2019 y del Presupuesto de Egresos de la Federación, años 2018 y 2019

Cuadro 3

Variaciones del Presupuesto de Instituciones Estatales y Fondos

Extraordinarios de Educación Superior, 2018-2019

(Millones de pesos)

\begin{tabular}{|c|c|c|c|c|c|c|}
\hline \multirow{2}{*}{$\begin{array}{l}\text { Programa } \\
\text { Presupuestario / } \\
\text { Unidad Responsable }\end{array}$} & \multirow{2}{*}{2018 a } & \multirow{2}{*}{$2019 p$} & \multirow{2}{*}{$\begin{array}{c}\text { Ampliaciones } \\
\text { Cámara de } \\
\text { Diputados }\end{array}$} & \multirow{2}{*}{2019 a } & \multicolumn{2}{|c|}{$\begin{array}{c}\text { Variación } 2018 \text { a - } \\
2019 \text { a }\end{array}$} \\
\hline & & & & & Absoluta & $\%$ real $^{1 /}$ \\
\hline $\begin{array}{l}\text { U006 Subsidios } \\
\text { para organismos } \\
\text { descentralizados } \\
\text { estatales }\end{array}$ & $66,308.5$ & $66,245.8$ & $2,672.0$ & $68,917.8$ & $2,609.3$ & $0.1 \%$ \\
\hline $\begin{array}{l}511 \text { Dirección General } \\
\text { de Educación Superior } \\
\text { Universitaria }\end{array}$ & $58,437.8$ & $58,647.9$ & n.d. & $61,019.2$ & $2,581.4$ & $0.5 \%$ \\
\hline
\end{tabular}




\begin{tabular}{|c|c|c|c|c|c|c|}
\hline \multirow{2}{*}{$\begin{array}{l}\text { Programa } \\
\text { Presupuestario / } \\
\text { Unidad Responsable }\end{array}$} & \multirow{2}{*}{2018 a } & \multirow{2}{*}{$2019 p$} & \multirow{2}{*}{$\begin{array}{l}\text { Ampliaciones } \\
\text { Cámara de } \\
\text { Diputados }\end{array}$} & \multirow{2}{*}{2019 a } & \multicolumn{2}{|c|}{$\begin{array}{c}\text { Variación } 2018 \text { a - } \\
2019 \text { a }\end{array}$} \\
\hline & & & & & Absoluta & $\%$ real $^{1 /}$ \\
\hline M00 TecNM & $3,472.8$ & $3,485.3$ & n.d. & $3,623.2$ & 150.4 & $0.5 \%$ \\
\hline $\begin{array}{l}\text { U079 - Expansión de } \\
\text { la Educación Media } \\
\text { Superior y Superior }\end{array}$ & 0.0 & 0.0 & 0.0 & 0.0 & 0.0 & $0.0 \%$ \\
\hline $\begin{array}{l}\text { U081 - Apoyos para la } \\
\text { Atención a Problemas } \\
\text { Estructurales de las upes }\end{array}$ & 700.0 & 0.0 & 0.0 & 0.0 & -700.0 & $-100.0 \%$ \\
\hline $\begin{array}{l}\text { S247 - Programa para el } \\
\text { Desarrollo Profesional } \\
\text { Docente }\end{array}$ & 656.4 & 246.6 & 0.0 & 246.6 & -409.8 & $-63.8 \%$ \\
\hline $\begin{array}{l}511 \text { Dirección General } \\
\text { de Educación Superior } \\
\text { Universitaria }\end{array}$ & 564.7 & 207.8 & 0.0 & 207.8 & -356.9 & $-64.6 \%$ \\
\hline M00 TecNM & 44.1 & 18.7 & 0.0 & 18.7 & -25.4 & $-59.2 \%$ \\
\hline $\begin{array}{l}514 \text { Coordinación } \\
\text { General de } \\
\text { Universidades } \\
\text { Tecnológicas y } \\
\text { Politécnicas }\end{array}$ & 47.6 & 20.2 & 0.0 & 20.2 & -27.5 & $-59.2 \%$ \\
\hline $\begin{array}{l}\text { S267 Fortalecimiento de } \\
\text { la Calidad Educativa }\end{array}$ & $1,862.6$ & $1,117.1$ & 0.0 & $1,117.1$ & -745.5 & $-42.3 \%$ \\
\hline $\begin{array}{l}511 \text { Dirección General } \\
\text { de Educación Superior } \\
\text { Universitaria }\end{array}$ & $1,104.6$ & 701.9 & 0.0 & 701.9 & -402.7 & $-38.8 \%$ \\
\hline $\begin{array}{l}514 \text { Coordinación } \\
\text { General de } \\
\text { Universidades } \\
\text { Tecnológicas y } \\
\text { Politécnicas } \\
\end{array}$ & 181.3 & 73.5 & 0.0 & 73.5 & -107.8 & $-61.0 \%$ \\
\hline $\begin{array}{l}515 \text { Dirección General } \\
\text { de Educación Superior } \\
\text { para Profesionales de la } \\
\text { Educación }\end{array}$ & 576.7 & 341.7 & 0.0 & 341.7 & -235.0 & $-43.0 \%$ \\
\hline $\begin{array}{l}\text { U040 Programa de } \\
\text { Carrera Docente (upes) }\end{array}$ & 350.0 & 354.9 & 0.0 & 354.9 & 4.9 & $-2.4 \%$ \\
\hline $\begin{array}{l}511 \text { Dirección General } \\
\text { de Educación Superior } \\
\text { Universitaria }\end{array}$ & 350.0 & 354.9 & 0.0 & 354.9 & 4.9 & $-2.4 \%$ \\
\hline $\begin{array}{l}\text { S244 Programa para la } \\
\text { Inclusión y la Equidad } \\
\text { Educativa }\end{array}$ & 52.5 & 37.8 & 0.0 & 37.8 & -14.7 & $-30.7 \%$ \\
\hline $\begin{array}{l}500 \text { Subsecretaría de } \\
\text { Educación Superior }\end{array}$ & 52.5 & 37.8 & 0.0 & 37.8 & -14.7 & $-30.7 \%$ \\
\hline $\begin{array}{l}\text { U080 Apoyos a centros } \\
\text { y organizaciones de } \\
\text { educación }\end{array}$ & 437.0 & 444.7 & 0.0 & 444.7 & 7.8 & $-2.0 \%$ \\
\hline $\begin{array}{l}500 \text { Subsecretaría de } \\
\text { Educación Superior }\end{array}$ & 27.4 & 27.8 & 0.0 & 27.8 & 0.5 & $-2.0 \%$ \\
\hline $\begin{array}{l}511 \text { Dirección General } \\
\text { de Educación Superior } \\
\text { Universitaria }\end{array}$ & 409.6 & 416.9 & 0.0 & 416.9 & 7.3 & $-2.0 \%$ \\
\hline
\end{tabular}




\begin{tabular}{|c|c|c|c|c|c|c|}
\hline \multirow{2}{*}{$\begin{array}{l}\text { Programa } \\
\text { Presupuestario / } \\
\text { Unidad Responsable }\end{array}$} & \multirow{2}{*}{2018 a } & \multirow{2}{*}{$2019 p$} & \multirow{2}{*}{$\begin{array}{l}\text { Ampliaciones } \\
\text { Cámara de } \\
\text { Diputados }\end{array}$} & \multirow{2}{*}{2019 a } & \multicolumn{2}{|c|}{$\begin{array}{c}\text { Variación } 2018 \text { a - } \\
2019 \text { a } \\
\end{array}$} \\
\hline & & & & & Absoluta & $\%$ real $^{1 /}$ \\
\hline $\begin{array}{l}500 \text { Subsecretaría de } \\
\text { Educación Superior }\end{array}$ & $5,169.3$ & $2,530.4$ & 0.0 & $2,530.4$ & $-2,638.9$ & $-52.9 \%$ \\
\hline $\begin{array}{l}515 \text { Dirección General } \\
\text { de Educación Superior } \\
\text { para Profesionales de la } \\
\text { Educación }\end{array}$ & 138.7 & 85.5 & 0.0 & 85.5 & -53.2 & $-40.7 \%$ \\
\hline $\begin{array}{l}\text { U083 Universidades } \\
\text { para el Bienestar Benito } \\
\text { Juárez García }\end{array}$ & 0.0 & $1,000.0$ & 0.0 & $1,000.0$ & $1,000.0$ & n.a. \\
\hline $\begin{array}{l}511 \text { Dirección General } \\
\text { de Educación Superior } \\
\text { Universitaria }\end{array}$ & 0.0 & $1,000.0$ & 0.0 & $1,000.0$ & $1,000.0$ & n.a. \\
\hline $\begin{array}{l}\text { U280 Jóvenes } \\
\text { Construyendo el Futuro }\end{array}$ & 0.0 & $4,320.0$ & 0.0 & $4,320.0$ & $4,320.0$ & n.a. \\
\hline $\begin{array}{l}500 \text { Subsecretaría de } \\
\text { Educación Superior }\end{array}$ & 0.0 & $4,320.0$ & 0.0 & $4,320.0$ & $4,320.0$ & n.a \\
\hline
\end{tabular}

1/ Crecimiento del Índice de precios implícitos del pib para 2019 de 3.9\%, de acuerdo con los Criterios Generales de Política Económica para la Iniciativa de Ley de Ingresos y el Proyecto de Presupuesto de Egresos de la Federación para el Ejercicio Fiscal 2019 .

a - aprobado.

$\mathrm{p}$ - proyecto.

n.a. - no aplica.

n.d. - no disponible.

Fuente: Elaboración propia con datos del Proyecto de Presupuesto de Egresos de la Federación 2019 y del Presupuesto de Egresos de la Federación, años 2018 y 2019.

\section{Retos de la universalización de la educación superior}

El presupuesto aprobado en educación superior, no obstante las ampliaciones obtenidas, presenta diferentes retos para las universidades y para el desarrollo de las políticas de universalización de la educación superior.

El 12 de mayo de 2018, en un acto de campaña en Guelatao, Oaxaca, López Obrador había dado a conocer los diez compromisos básicos con la educación y con el magisterio nacional; tres referidos a educación superior. ${ }^{12}$ Uno de ellos estaba directamente relacionado con la propuesta de cancela-

${ }^{12}$ La incorporación de los jóvenes a educación superior formó parte de la plataforma electoral de Morena presentada en el documento Proyecto Alternativo de Nación 20182024: “La exclusión de millones de jóvenes de oportunidades educativas ha sido una de las causas preponderantes de la descomposición social que padece el país. Preferimos que los jóvenes sean becarios a que sean sicarios. Por ello, habrá acceso a escuelas universitarias públicas y privadas para todos los estudiantes que aspiren a estudiar y cuenten con certificado de estudios de educación media superior". (Morena, 2018: 23). 
ción de la Reforma Educativa de 2013, principal compromiso político ante el magisterio nacional en general y con la Sección 22 de la CNTE de Oaxaca en particular. Se propuso el fortalecimiento de las Escuelas Normales y de la Universidad Pedagógica Nacional, por el rol que jugarían en la renovación de la formación del magisterio y la elaboración del nuevo plan educativo que se desprendería de la modificación al artículo $3^{\circ}$ constitucional y de las leyes secundarias.

También se formuló el compromiso de que los estudiantes de familias de escasos recursos económicos que estudien en universidades o en escuelas de nivel superior obtendrían una beca, y de que no habría rechazados en las universidades, lo que se tradujo en nuevos instrumentos de política: el programa de becas Jóvenes Escribiendo el Futuro, que es el componente en la SEP del programa de capacitación Jóvenes Construyendo el Futuro de la Secretaría del Trabajo y Previsión Social. La meta fue otorgar 300 mil becas de 2,400 pesos mensuales a estudiantes de educación superior de escasos recursos, dando prioridad a estudiantes indígenas, afrodescendientes y que vivan en zonas de pobreza o de violencia. Este nuevo programa de becas coexistiría con las becas de manutención (antes becas PRONABES), que otorgan menores montos a los estudiante (hasta 9 mil pesos al año).

El compromiso de no rechazo a los aspirantes a ingresar a educación superior estuvo asociado a la extensión de la gratuidad educativa. De acuerdo con el discurso del Presidente en la ceremonia de presentación del programa de becas antes referido, el ingreso universal a educación superior sería posible si el ingreso a este nivel educativo se consideraba como un derecho universal del pueblo, y no como el privilegio de unos cuantos. Se refirió a que los exámenes de admisión servían de pretexto para rechazar cada año a 300 mil jóvenes, y ocultar la falta de espacios ante la falta de inversión y de presupuesto durante el periodo neoliberal (López Obrador, 2019). En esa alocución no se refirió al hecho de que son las universidades autónomas y las instituciones públicas de educación superior las que fijan los criterios de selección e ingreso de los estudiantes, y que el establecimiento de las políticas institucionales es atribución de sus órganos de gobierno.

Desde la campaña presidencial se anunció que la obligatoriedad y la gratuidad de la educación se harían extensivas al nivel superior. Con este propósito, el senador Martí Batres de Morena, y primer presidente de la mesa directiva de la Cámara de Senadores, a los pocos días de instalada la XLIV Legislatura presentó una iniciativa de reforma al artículo tercero constitucional en la que se establecía la obligatoriedad de la educación superior. Posteriormente, el 12 de diciembre, se presentó la iniciativa presidencial con proyecto de decreto que reforma los artículos 3, 31 y 73 de la Constitución 
Política de los Estados Unidos Mexicanos la cual, además de los cambios a la reforma educativa del gobierno de Peña Nieto, incorporó la obligatoriedad, la gratuidad y la universalización de la educación superior. Como resultado de un complicado proceso de negociaciones a lo largo de cinco meses entre la SEP, el grupo de Morena, los grupos de oposición y la Coordinadora Nacional de Trabajadores de la Educación (CNTE), y una vez que se contó con la aprobación de la mayoría de las legislaturas locales, el 15 de mayo se publicó el decreto que contiene las nuevas disposiciones constitucionales en materia educativa, entre las cuales está la obligatoriedad de la educación superior (Poder Ejecutivo, 2019).

La iniciativa de reforma del Ejecutivo señalaba que el Estado promovería la inclusión de los jóvenes a la educación superior y establecería políticas enfocadas a evitar la deserción y fomentar su permanencia. En el decreto de reformas se acordó una nueva disposición (Fracción x) en los siguientes términos:

La obligatoriedad de la educación superior corresponde al Estado. Las autoridades federal y locales establecerán políticas para fomentar la inclusión, la permanencia y la continuidad, en términos que la ley señale. Asimismo, proporcionarán medios de acceso a este tipo educativo para las personas que cumplan con los requisitos dispuestos por las instituciones públicas.

El compromiso presidencial de cero rechazados y el establecimiento de la obligatoriedad de la educación superior estuvieron asociados a la creación de nuevas universidades gratuitas y sin exámenes de selección en zonas marginadas del país. El programa fue perfilándose como uno de los prioritarios del gobierno federal para la expansión de la oferta en el nivel superior, y en el PEF se estableció su denominación oficial: Universidades para el Bienestar Benito Juárez García, con sede en el Centro de Cooperación Regional para la Educación de Adultos en América Latina y el Caribe (CREFAL, 2019), en la ciudad de Pátzcuaro, Michoacán. Hasta el mes de mayo de 2019, se contaba con 83 sedes abiertas que atendían a cerca de 8,000 estudiantes y cerca de 5,000 profesores. Se dijo que conforme se avanzara en la habilitación de las sedes y los acuerdos con las comunidades se iría ampliando la población atendida, para llegar al fin del sexenio a atender a 300 mil estudiantes. En la mayoría de los nuevos establecimientos se ofrecería un programa educativo que estuviera relacionado con las necesidades de formación de profesionales para el desarrollo local (crefal, 2019). Las universidades que en años anteriores había impulsado Morena pasarían a formar parte del nuevo sistema universitario, proceso que no estaría exento de conflictos.

Los programas de becas y de nuevas universidades respondían a la estrategia del nuevo gobierno de impulsar programas sociales dirigidos a la po- 
blación de mayores exclusión, pobreza o vulnerabilidad, y que contribuyen al aumento del acceso efectivo de los derechos sociales, respondiendo a los criterios que se establecieron desde la transición.

Sin embargo, los ajustes a la baja en los fondos extraordinarios y el estancamiento del subsidio ordinario de las universidades existentes jugaba en contra del propósito de ampliación de la cobertura para alcanzar la universalización de la educación superior, además de que se frenarían acciones para la reestructuración financiera de las universidades en situación de crisis recurrente. Con los datos de matrícula de las nuevas universidades en el primer semestre de 2019, se apreciaba que tendría una baja contribución a la ampliación de la cobertura en el sexenio.

\section{Ampliación de la cobertura}

En el Programa Sectorial de Educación 2013-2018 se establecieron dos metas de cobertura de educación superior para el fin de ese sexenio: 1. Tasa bruta de escolarización de $40 \%$ con paridad entre hombres y mujeres; y 2. Tasa bruta de escolarización de $17 \%$ para los primeros cuatro deciles de ingresos monetarios per capita. De acuerdo con la información del Formato 911 del ciclo escolar 2018-2019, la matrícula de técnico superior universitario y de licenciatura, en las modalidades escolarizada y no escolariza, ascendió a 4,344,644. Con las estimaciones actualizadas por CONAPO del grupo 18-22 años, la tasa bruta de escolarización fue del 39.7\%, tan sólo tres décimas por debajo de la meta establecida por el gobierno de Peña Nieto.

$\mathrm{Al}$ inicio de la nueva administración de la SEP se contaba con dos referentes para el establecimiento de metas de cobertura de educación superior. ${ }^{13}$ El primero de ellos era la meta propuesta por la ANUIES en el documento "Visión y Acción 2030" entregado a los candidatos a la Presidencia en mayo de 2018 y a López Obrador en su calidad de Presidente electo. La meta de 50\% de cobertura propuesta por la ANUIES implicaría incrementar la matrícula en el período 2019-2024 1.13 millones de estudiantes, con un porcentaje de variación de $26 \%$. Para alcanzar esta meta, cada año se tendrían que incorporar 189 mil estudiantes de técnico superior universitario y de licenciatura. En

\footnotetext{
${ }^{13} \mathrm{Al}$ momento de escribir el artículo aún no se daban a conocer las metas de cobertura. El 30 de abril de 2019 el Presidente sometió a consideración de la Cámara de Diputados el Plan Nacional de Desarrollo 2019-2024 (Presidencia de la República, 2019), en cuyo texto no se establecen metas en este indicador, las que eventualmente serían consideradas en el Programa Sectorial de Educación.
} 
el supuesto de que la participación relativa de la matrícula por régimen de sostenimiento se mantuviera sin variación en el período (67\% pública y $33 \%$ privada), la matrícula de las instituciones públicas se incrementaría en 126 mil estudiantes cada año, no obstante lo cual, de acuerdo con las proyecciones de la SEP, no se tendrían lugares para todos los egresados de bachillerato al finalizar el sexenio.

Un segundo ejercicio, elaborado por los equipos técnicos de la Subsecretaría de Educación Superior, consideró que para que la totalidad de egresados de bachillerato tuviera un lugar en educación superior se tendría que lograr una cobertura de $56 \%$ en 2024. Ello requeriría aumentar la matrícula durante el sexenio en 41\% para alcanzar 6.13 millones de estudiantes en 2024 . Anualmente se tendrían que aumentar 298 mil lugares, de los cuales 200 mil serían en el segmento público.

Para ponderar cada uno de los escenarios esbozados, es ilustrativa la comparación con lo avanzado en los dos sexenios anteriores. En el de Felipe Calderón la cobertura pasó del 25.1 al 31.7\%, con un promedio anual de variación de la matrícula pública de 5.7\%, (112 mil nuevos lugares). En el sexenio de Peña Nieto la cobertura pasó del 37.1 al 39.7\% y la variación anual de la matrícula pública fue de 3.5\% (89 mil lugares).

El escenario de 50\% de cobertura para 2024 implicaría, en términos absolutos, un esfuerzo mayor que el realizado en los dos sexenios anteriores: se requeriría abrir 14 mil lugares más de los logrados en el sexenio de Calderón y 38 mil más respecto del sexenio de Peña Nieto. Sin embargo, en términos relativos, la variación porcentual sería de casi dos puntos porcentuales menos que en el sexenio de Calderón. Por el contrario, el escenario de 56\% de cobertura implicaría una variación anual de la matrícula pública mayor en 1.4 puntos porcentuales a las de ese sexenio y del doble de la de Peña Nieto.

Los avances logrados en la ampliación de la cobertura de los dos sexenios previos fue posible por la existencia de recursos públicos destinados a tal propósito: el Fondo de Aportaciones Múltiples que destina recursos para infraestructura (situado en el Ramo 33 Aportaciones Federales para Entidades Federativas y Municipios), y los programas que con distintas denominaciones administró la SEP para la ampliación de la cobertura. ${ }^{14}$ En estos últimos

${ }^{14}$ De 2006 a 2019 se contó con los siguientes fondos para ampliación de cobertura en educación superior: Fondo para incremento de la matrícula en educación superior de las Universidades Públicas Estatales y con Apoyo Solidario (2007 a 2010); Fondo para ampliación de la oferta educativa de nivel superior (2008 a 2010); Fondo para ampliar y diversificar la oferta educativa en educación superior (2011 a 2013); Fondo para ampliación de la oferta educativa en los Institutos Tecnológicos (2008 a 2013); Fondo de apoyo a la infraestructura de las universidades interculturales (2008 a 2013); Programa 
programas, de 2008 a 2015, año en que iniciaron los ajustes presupuestales, se destinaron en promedio anual 1,800 mdp para proyectos de expansión de la oferta educativa. Con estos recursos se crearon nuevas universidades, 140 en el sexenio de Calderón y 26 en el de Peña Nieto (Mendoza, 2017), en tanto se ampliaron las existentes con el avance en la desconcentraron territorial de su oferta educativa.

El reto que se presenta en el nuevo escenario presupuestario es la obtención de recursos para la expansión de la matrícula en los distintos subsistemas de educación superior: el sistema de Universidades para el Bienestar Benito Juárez García cuenta con un presupuesto de 1,000 mdp para la construcción, el equipamiento y la operación de los cien planteles programados (si se promedia el presupuesto aprobado, cada uno contaría con $10 \mathrm{mdp}$ ); las universidades públicas estatales, con apoyo solidario, interculturales, tecnológicas y politécnicas, así como los institutos tecnológicos del Tecnológico Nacional de México y las escuelas normales no cuentan con presupuesto etiquetado para la ampliación de su oferta educativa; el programa presupuestario U079, Expansión de la educación media superior y superior, asignó solamente 116 mdp en 2017 y no contó con presupuesto en 2018 y 2019. La inexistencia de recursos focalizados a la ampliación de la cobertura será uno de los principales desafíos que enfrentará la política de acceso universal, obligatoriedad y gratuidad de la educación superior de la nueva administración.

En el período de elaboración del PEF se advirtió a las autoridades hacendarias, por parte del equipo de transición de la SEP, que la omisión de recursos para la expansión de la oferta en instituciones existentes contravenía el compromiso de acceso universal a educación superior y dificultaría el establecimiento de acuerdos con las universidades para su expansión futura.

Estimaciones preliminares de la Subsecretaría de Educación Superior sobre los costos de la ampliación de la cobertura en el sexenio 2012-2018, tanto para inversión como para la operación regular de las instituciones educativas, oscilaban entre poco más de 6 mil y 10 mil mdp anuales, ${ }^{15}$ de acuerdo con los diversos escenarios planteados hasta el primer trimestre de 2019, y solamente se disponía de los recursos del Fondo de Aportaciones Mútiples, del orden de los 4,500 mdp, y de los recursos asignados a las cien nuevas universidades (1,000 mdp). Frente a este panorama, las autoridades educativas

de Expansión en la Oferta Educativa en Educación Media Superior y Superior (2014, 2015); Expansión de la educación media superior y superior (2016 y 2017).

${ }^{15}$ Rodolfo Tuirán, quien ocupó la titularidad de las subsecretarías de educación media superior y de educación superior en la administración de Peña Nieto, en un artículo publicado en la revista Nexos presentó estimaciones similares sobre los costos que tendría la obligatoriedad y la gratuidad de la educación superior (Tuirán, 2019). 
señalaron que se podría incrementar la matrícula con el aprovechamiento óptimo de la capacidad instalada de las IES.

\section{Gratuidad de la educación superior}

Un segundo desafío es la gratuidad de la educación superior que se desprende de su obligatoriedad. El artículo décimo cuarto transitorio del decreto señala que la legislación secundaria determinará la gradualidad para la implementación de los cambios y que la Cámara de Diputados, en el Presupuesto de Egresos de la Federación, aprobará las previsiones presupuestarias necesarias para su cumplimiento progresivo. Además, el artículo décimo quinto transitorio establece que para dar cumplimiento al principio de obligatoriedad de la educación superior, "[...] se incluirían los recursos necesarios en los presupuestos federal, de las entidades federativas y de los municipios $[\ldots]$ ", y que adicionalmente se establecerá un fondo federal especial que "[...] asegure a largo plazo los recursos económicos necesarios para garantizar la obligatoriedad de los servicios [...]", así como la plurianualidad de la infraestructura (Poder Ejecutivo, 2019).

Estimaciones realizadas por la ANUIES y por la Subsecretaría de Educación Superior sobre los impactos presupuestales de la gratuidad manejaron cifras cercanas a los 12 mil millones de pesos anuales, monto que equivale a los ingresos que obtienen las IES públicas por concepto de inscripción y reinscripción de alumnos. De acuerdo con el artículo décimo cuarto transitorio del decreto de reforma constitucional, los montos anuales necesarios, según la gradualidad en la implantación de esta disposición, deberían estar considerados en el presupuesto de las instituciones.

Un antecedente sobre la implantación de la gratuidad de la educación superior a nivel estatal es el caso de la Universidad Michoacana de San Nicolás de Hidalgo (UMSNH). En 2010 se modificó la Constitución Política del Estado de Michoacán, en la que se estableció que el Estado y los municipios están obligados a impartir educación preescolar, primaria, secundaria, media superior y superior, y que toda educación que el Estado impartiera será gratuita. Esta disposición sería gradual en los siguientes términos: "[...] inicialmente la gratuidad implicará el pago de inscripción en las instituciones públicas de media superior y superior hasta el grado de licenciatura, otros servicios como exámenes, cursos, certificados, credenciales, cartas de pasantía, titulación y constancias, se otorgarán de acuerdo a la disponibilidad presupuestal del Estado" (Poder Ejecutivo del Estado de Michoacán, 2010). 
Para dar cumplimiento a la disposición constitucional, se publicaron "lineamientos para el otorgamiento de apoyos para las instituciones públicas de educación media superior y superior para garantizar la obligatoriedad y la gratuidad" (Poder Ejecutivo del Estado de Michoacán, 2011), con los cuales se otorgarían los recursos que las instituciones percibían por concepto de inscripciones y reinscripciones. De acuerdo con información de la UMSNH, solamente en cuatro años el gobierno del estado asignó recursos por gratuidad (50 mdp en 2010, 43 mdp en 2011, 80 mdp en 2012 y 8 mdp en 2013). Ante el incumplimiento del compromiso del gobierno del estado, en 2014 el Consejo Universitario acordó cobrar nuevamente cuotas de inscripción y reinscripción a los alumnos. Una estudiante solicitó un amparo por considerar que se violaba su derecho a la educación, caso que fue atraído por la Suprema Corte de Justicia de la Nación, cuya resolución fue favorable a la quejosa, con el argumento de la progresividad de los derechos humanos y la consecuente obligación de implantar gradualmente la educación superior gratuita establecida en la Constitución local. ${ }^{16}$

Este caso ilustra los retos presupuestales que implica la gratuidad de la educación superior. En un escenario de creciente presión de las finanzas públicas y de disminución del gasto destinado a educación, la gratuidad, sin la correspondiente asignación de recursos crecientes por parte de la federación y de los estados, constituiría una presión adicional a la situación financiera por la que atraviesan las instituciones públicas de educación superior del país. La incorporación en el decreto de reforma constitucional de los artículos transitorios ya referidos evitará esta situación al obligar a las autoridades hacendarias de los diferentes niveles de gobierno a considerar los recursos necesarios en los presupuestos anuales de egresos.

\section{Sostenibilidad financiera de las universidades públicas}

Como hemos visto, las negociaciones para el presupuesto de educación superior realizadas en el último trimestre de 2018 estuvieron inmersas en un contexto de crítica y cuestionamiento a la universidad pública. Diputados y senadores de Morena de la nueva legislatura se referían, al igual que diversas expresiones de las redes sociales, a la opacidad en las finanzas uni-

\footnotetext{
${ }^{16}$ La Suprema Corte de Justicia de la Nación concluyó que la Universidad había violado, en perjuicio de la quejosa, el derecho a la educación superior gratuita, por lo que por unanimidad de cuatro votos confirmó la sentencia de primera instancia y concedió el amparo para el efecto de que no se le cobren las cuotas de inscripción durante su educación superior (Soberanes, 2018).
} 
versitarias; a los elevados salarios del personal académico de carrera y de la alta burocracia universitaria y a la existencia de actos de corrupción. A este ambiente de cuestionamiento se aunó la omisión de la fracción VII en la iniciativa del Ejecutivo de reforma al artículo tercero constitucional, ${ }^{17}$ lo cual llevó a pronunciamientos de las universidades sobre la defensa de la autonomía universitaria. Esta omisión, sumada a los "errores de cálculo" del presupuesto para educación superior, como fue reconocido en su momento por las autoridades hacendarias y por el Presidente, contribuyó a crear un clima de incertidumbre sobre la política que seguiría la nueva administración para las universidades públicas.

Adicionalmente, dos elementos abonaron a este clima. Por una parte, la Unidad de Inteligencia Financiera de la sHCP dio a conocer en el mes de febrero que se bloquearon las cuentas de una universidad pública estatal por la realización de depósitos y transferencias bancarias inusuales provenientes de más de 22 países, habiéndose detectado que se recibieron 150 millones de dólares provenientes de cuentas bancarias ubicadas en Suiza (SHCP, 2019a). Este comunicado, sin decir el nombre de la universidad, sembró nuevas dudas sobre la transparencia financiera de todas las universidades públicas. A los pocos días se informó que la universidad en cuestión era la Universidad Autónoma del Estado de Hidalgo. ${ }^{18}$ Por otra parte, la autonomía universitaria volvió a situarse en el debate público a principios de abril de 2019 por la presentación de iniciativas de modificación de las leyes de las universidades autónomas de Baja California Sur y del Estado de México, sin haberse contado con la participación de las comunidades universitarias respectivas.

Lo anterior se aunó a las medidas de austeridad establecidas en el decreto de PEF 2019, a las disposiciones contenidas en la Ley Federal de Austeridad Republicana - aprobada por la Cámara de Diputados en el mes de abril - y a las instrucciones dadas por el Presidente por medio de un memorandum, que implicaban recortes adicionales del $30 \%$ en diversas partidas del gasto operativo de las dependencias de la administración pública federal, con afectaciones a diversos organismos educativos y de investigación. ${ }^{19}$ En reacción a ello, di-

\footnotetext{
${ }^{17}$ El Secretario de Educación Pública, Esteban Moctezuma, señaló que la omisión de la fracción VII sobre la autonomía universitaria había sido un error que se corregiría en el proceso legislativo, tal como ocurrió durante la elaboración del dictamen correspondiente.

${ }^{18}$ El rector de la Universidad Autónoma del Estado de Hidalgo solicitó un amparo contra el congelamiento de las cuentas bancarias y rechazó que la universidad hubiera recibido recursos de procedencia ilícita o del extranjero. Reconoció que los recursos transferidos correspondían a los fondos de pensiones de los trabajadores.

${ }^{19}$ Con el memorándum del Presidente del 3 de mayo de 2019 se afectaban, entre otras instituciones los Centros Públicos de Investigación CONACYT, Centro de Investigación y
} 
versas organizaciones académicas y científicas manifestaron que la aplicación indiscriminada de estas medidas afectaría la realización de actividades académicas y de investigación en el país.

El cuestionamiento a la universidad pública por el manejo de los recursos, las medidas de austeridad y los problemas que de manera histórica han enfrentado en materia financiera, plantean con mayor urgencia la necesidad de revisar las políticas vigentes. La ANUIES ha señalado de manera reiterada que el esquema vigente de financiamiento presenta diversos problemas para el desarrollo de sus actividades sustantivas. Las principales propuestas presentadas desde el año 2000 han apuntado a incrementar los presupuestos federal y estatales para dar suficiencia financiera a todas las IES públicas, además de modificar la legislación educativa y presupuestaria para dar certeza sobre la disponibilidad de recursos con una visión plurianual.

El proceso de negociación del presupuesto para 2019 se dio en paralelo a la gestión de recursos para que nueve universidades públicas estatales pudieran cerrar el año y cumplir con el pago de salarios a sus trabajadores. ${ }^{20} \mathrm{La}$ crisis financiera fue resultado de diversos factores, entre ellos: contratación de personal académico y administrativo ante la política de incremento de la cobertura y expansión de la oferta educativa; insuficiencia del subsidio público para el pago del salario y de las prestaciones pactados en los contratos colectivos de trabajo; adeudos fiscales y a instituciones de seguridad social, y peso financiero de los sistemas de pensiones y jubilaciones. A ello hay que sumar la falta de compromiso de muchos gobiernos estatales para asignar a las universidades, en tiempo y forma, el presupuesto establecido en el convenio de apoyo financiero.

Desde el gobierno anterior se venían sosteniendo reuniones de trabajo entre las universidades, la SEP y la SHCP, sin que se hubieran logrado acuerdos para el rescate financiero; a diferencia de ello, con las autoridades del gobierno entrante se logró la asignación de recursos extraordinarios no regularizables por un monto de 2,300 mdp la cual, y en palabras del Presidente, no se repetiría en los siguientes años de su gobierno. La asignación de los re-

Estudios Avanzados, Instituto Politécnico Nacional, Tecnológico Nacional de México, El Colegio de México, además de los Institutos de Salud.

${ }^{20}$ Las universidades apoyadas fueron: Universidad Autónoma de Chiapas, Universidad Benito Juárez Autónoma de Oaxaca, Universidad Juárez Autónoma de Tabasco, Universidad Autónoma del Estado de México, Universidad Autónoma del Estado de Morelos, Universidad Michoacana de San Nicolás de Hidalgo, Universidad Autónoma de Nayarit, Universidad Autónoma de Sinaloa y Universidad Autónoma de Zacatecas. Adicionalmente, la Universidad Veracruzana venía solicitando apoyo para cubrir las deudas fiscales con el SAT en que había incurrido el gobierno de Javier Duarte. 
cursos estuvo condicionada al establecimiento de compromisos de disciplina financiera por parte de las universidades, con el seguimiento, por parte de la Dirección General de Educación Superior Universitaria, a través de un portal de acceso abierto (sep.entransparencia.mx). ${ }^{21}$

En el primer trimestre del año se realizó un diagnóstico de la situación financiera de las nueve universidades, con el reconocimiento de que el déficit financiero no era un problema circunscrito a ellas, sino que se hacía extensivo a otras instituciones públicas de educación superior, tanto federales como estatales. Los problemas de sostenibilidad financiera se verían agravados por la inexistencia de recursos en el programa presupuestario U081 Apoyos para la atención a problemas estructurales de las UPES, que en años anteriores se habían destinado a la capitalización de los fondos de pensiones, al reconocimiento de plantilla y al pago de adeudos al sat y a instituciones de seguridad social. El no contar con recursos adicionales para estos propósitos pondría en mayor riesgo la viabilidad financiera de las universidades.

\section{Conclusiones}

De la revisión del proceso de formulación del presupuesto de educación superior para 2019 se pueden formular algunas conclusiones:

1. En primer término, el proceso de formulación, negociación y aprobación del presupuesto federal para educación superior adquirió características inéditas, no sólo por tratarse del diseño presupuestal para el primer año de una nueva administración, sino por las prioridades políticas de un gobierno que se propone la "cuarta transformación" del país, la modificación de las políticas impulsadas en México desde los años ochenta del siglo pasado (gobiernos neoliberales), y el establecimiento de nuevos programas de gobierno.

2. El proceso presupuestal estuvo orientado por los lineamientos políticos establecidos en los documentos de Morena y los posicionamientos de An-

${ }^{21}$ En los convenios que se firmaron entre la SEP, el gobierno estatal y la universidad, se establecieron medidas de orden general para la disciplina financiera, y un anexo de ejecución para cada universidad con la elaboración de un plan de acción con compromisos y medidas específicas para atender la problemática financiera. En los términos del convenio, las universidades se comprometieron, entre otras cosas, a "asegurar que, para la atención al déficit financiero, se incorporen o fortalezcan en su reglamentación las disposiciones en materia de disciplina financiera institucional, observando los principios de legalidad, honestidad, eficacia, eficiencia, economía, racionalidad, austeridad, transparencia, control y rendición de cuentas en el ejercicio de los recursos públicos" (DGESU, 2019). 
drés Manuel López Obrador, primero como candidato y después como Presidente. Entre ellos, destacó el discurso de austeridad y de combate a la corrupción, con la premisa de que, para el impulso de la transformación del país, se requería una cirugía mayor del PEF y acabar con el manejo discrecional de los recursos públicos en los diferentes niveles de la administración pública federal.

3. Durante el proceso de diseño presupuestal, las universidades e instituciones públicas de educación superior fueron objeto, por parte de diversos funcionarios y legisladores del grupo político mayoritario, de señalamientos de privilegio, corrupción y opacidad en el ejercicio de los recursos. El tema de la autonomía universitaria, y su omisión en la iniciativa presidencial de reforma del artículo tercero constitucional, formó parte del entramado político en el que se decidieron las asignaciones presupuestales.

4. El énfasis en la formulación del presupuesto estuvo puesto en los programas sociales con beneficiarios directos y sin intermediación de instituciones. Ello se reflejó en el presupuesto de educación superior con la introducción de dos nuevos programas presupuestarios dirigidos a los grupos de población de menores ingresos: Jóvenes Construyendo el Futuro y Universidades para el Bienestar Benito Juárez García.

5. Como resultado de las presiones ejercidas por los rectores de las universidades públicas, por la ANUIES y por otros actores educativos, no avanzaron las propuestas iniciales presentadas por las autoridades hacendarias de reducción en el presupuesto ordinario de las Instituciones de Educación Superior. A diferencia de ello, los fondos extraordinarios tuvieron recortes significativos, con lo que se dio continuidad a la tendencia de reducción presupuestal observada en la segunda mitad del gobierno de Enrique Peña Nieto. La disminución de recursos destinados a los fondos extraordinarios dificultará la realización de proyectos de las universidades tanto para la ampliación de la cobertura como para el saneamiento financiero.

6. El resultado de las negociaciones presupuestales para el primer año del gobierno de Andrés Manuel López Obrador refuerza la petición formulada por las universidades públicas y por la ANUIEs de reformular el modelo de financiamiento de la educación superior para dar suficiencia y certeza presupuestales a todas las instituciones públicas con visión de largo plazo. La obligatoriedad, la gratuidad y la universalización de la educación superior establecidas en el artículo tercero constitucional requerirán de una nueva política de financiamiento, así como de un nuevo pacto entre el gobierno federal y los gobiernos estatales, que dé viabilidad financiera a todas las instituciones públicas de educación superior.

\section{Referencias}

ANUIES (2018a). Visión y Acción 2030. Propuesta de la ANUIES para renovar la Educación Superior en México. México: Autor. 
ANUIES (2018b). Desplegado 17 de diciembre de 2018. Recuperado el 10 de enero de 2019, de http://www.anuies.mx/media/docs/avisos/pdf/1812171934462019.pdf

Casar, María Amparo. (2001). El proceso de negociación presupuestal en el primer gobierno sin mayoría: un estudio de caso. México: CIDE.

Comisión de Presupuesto y Cuenta Pública de la Cámara de Diputados (2018). Dictamen con Proyecto de Decreto de Presupuesto de Egresos de la Federación para el Ejercicio Fiscal 2019. Gaceta Parlamentaria, Año xxii, Núm. 5186A, 23 de diciembre de 2018. Recuperado el 17 de enero de 2019, de http:/ / gaceta.diputados.gob.mx/PDF/64/2018/dic/20181223-A.pdf

CONEVAL (2018). Consideraciones para el proceso presupuestario 2019, 1 Junio 2018. Recuperado el 5 de febrero de 2019, de https:/ / www.coneval.org.mx/ Evaluacion/IEPSM/Documents/Consideraciones-2019.pdf

CREFAL (2019). Portal universidades para el Bienestar Benito Juárez García. https://www.crefal.org/ universidades

Dávila Estefan, David y Lila Caballero Sosa (2005). El sistema de comisiones, el cabildeo legislativo y la participación ciudadana en el Congreso mexicano, Cuadernos del seguimiento ciudadano al Poder Legislativo en México, México, Fundar, Centro de Análisis e Investigación.

DGESU (2019). Dirección General de Educación Superior Universitaria. Portal de transparencia, https:/ / sep.entransparencia.mx

El Heraldo de México (17 de diciembre 2018). "Carlos Urzúa defiende recorte presupuestal a universidades". Recuperado el 5 de enero de 2019, de https:/ / heraldodemexico.com.mx/mer-k-2/carlos-urzua-defiende-recorte-presupuestal-a-universidades/

El Heraldo de México (28 de mayo de 2019. "Universidades Benito Juárez ya cuentan con 83 planteles, aún faltan 18: Raquel Sosa". Recuperado el 30 de mayo de 2019 de https:/ / heraldodemexico.com.mx/pais/universidades-benito-juarez-ya-cuentan-con-83-planteles-aun-faltan-18-raquel-sosa/

El Financiero (17 diciembre 2018). " unam cuestiona reducción en su presupuesto que propone el Gobierno de amlo". Recuperado el 20 de diciembre de 2018, de file:///G:/A\%20AAA \%20ARTÍCULO\%20pef\%202019\%20MARZO\%20 19 / unam \% 20cuestiona \% 20reducción \% 20en \% 20su \% 20presupuesto \% 20 que $\% 20$ propone $\% 20 \mathrm{el} \% 20$ Gobierno $\% 20 \mathrm{de} \% 20 \mathrm{AMLO}$.html

Galaviz Efrén, Elías (2006). El cabildeo legislativo y su regulación, Serie Estudios Jurídicos, núm. 101, Instituto de Investigaciones Jurídicas, México, UNAM.

Heredia Sánchez, Edgar D. (2004). “Cabildeo. Nuevos instrumentos de gobernanza democrática", en Revista Casa del Tiempo, Junio de 2004, México, UAM.

Hernández, Georgina (19 diciembre 2018). “Así es como amlo compensará el "error" del recorte al presupuesto de universidades". HuffPost México. Recuperado el 8 de abril de 2019, de https:/ / es-us.noticias.yahoo.com/amlocompensar\%C3\% A1-error-recorte-presupuesto-160500920.html

Jiménez, Néstor (18 diciembre 2018). “López Obrador defiende el presupuesto: haremos más con menos". La Jornada. Recuperado el 26 de abril de 2019, de https://www.jornada.com.mx/2018/12/18/politica/004n2pol

Lerdo de Tejada, Sebastián y Luis Antonio Gomina (2004). El Lobbying en México. México, Miguel Ángel Porrúa. 
López Obrador, Andrés Manuel (2019). Versión estenográfica. “Becas 'Jóvenes Escribiendo el Futuro' desde la Plaza de las Tres Culturas, Ciudad de México". Recuperado el 10 de abril de 2019, de https://lopezobrador.org. $\mathrm{mx} / 2019 / 02 / 12 /$ version-estenografica-becas-jovenes-escribiendo-el-futurodesde-la-plaza-de-las-tres-culturas-ciudad-de-mexico /

Mendoza Rojas, Javier (2007). Cabildeo legislativo para el presupuesto federal de educación superior. Papel de la ANUIES y resultados en el periodo 20012007. México, IISUE-UNAM - ANUIES.

Mendoza Rojas, Javier (2017). "Financiamiento de la educación superior en la primera mitad del gobierno de Enrique Peña Nieto: ¿fin del periodo de expansión?", en Perfiles Educativos, México, vol. xxxix, núm. 156, 3a. época, abril-junio 2017, pp. 119-140, IISUE-unam.

Mendoza Rojas, Javier (2018). “Situación y retos de la cobertura del sistema educativo nacional", en Perfiles Educativos, México, vol. XL, número especial, 2018, pp. 11-52, IISUE-unam.

Minuta por el que se reforman, adicionan y derogan diversas disposiciones de los artículos 3o., 31 y 73 de la Constitución Política de los Estados Unidos Mexicanos, en materia educativa., Gaceta del Senado del día Jueves 25 de abril de 2019. Gaceta: LXIV/1SPO-128/94829. Rescatada el 29 de abril de 209, de http://www.senado.gob.mx/64/gaceta_del_senado/documento/94829, consultado el 29 de abril de 2019.

Morena (2018). Proyecto Alternativo de Nación 2018-2024. Plataforma Electoral y Programa de Gobierno. Recuperado el 29 de abril de 2019, de https:/ / repositoriodocumental.ine.mx/xmlui/bitstream/handle/123456789/94946/ CGor201801-31-ap-20-8-a1.pdf

Nación 321 (2018). "amlo sobre recortes en el Presupuesto: se va a hacer más con menos". Recuperado el 15 de marzo de 2019, de https:/ / www.nacion321.com/ gobierno/amlo-sobre-recortes-en-el-presupuesto-se-va-a-hacer-mas-con-menos

Poder Ejecutivo, Secretaría de Gobernación (15 de mayo de 2019). Decreto por el que se reforman, adicionan y derogan diversas disposiciones de los artículos 3o. 31 y 73 de la Constitución Política de los Estados Unidos Mexicanos, en materia educativa, Diario Oficial de la Federación, 15 de mayo de 2019 (edición vespertina). Recuperado el 20 de mayo de 2019, de https://www.dof. gob.mx/index.php?year=2019\&month $=05 \&$ day $=15$

Poder Ejecutivo del Estado de Michoacán (2010). Decreto por el que se reforman y adicionan los artículos 138 y 139 de la Constitución Política del Estado Libre y Soberano de Michoacán de Ocampo. Periódico Oficial del Gobierno Constitucional del Estado de Michoacán de Ocampo. Tomo cxlix, Núm. 69, 6 de agosto de 2010. Recuperado el 6 de febrero de 2019, de http:/ / leyes.michoacan.gob.mx/destino/O4764po.pdf

Poder Ejecutivo del Estado de Michoacán (2011). “Lineamientos para el otorgamiento de apoyos para las instituciones públicas de educación media superior y superior para garantizar la obligatoriedad y gratuidad". Periódico Oficial del Gobierno Constitucional del Estado de Michoacán de Ocampo, Tomo cli, Núm. 95, 20 de junio de 2011). Recuperado el 6 de febrero de 2019, de http:/ / www.ordenjuridico.gob.mx/Documentos/Estatal/Michoacan/wo64110.pdf 
Presidencia de la República (2019). Plan Nacional de Desarrollo 2019-2024, en Gaceta Parlamentaria, año xxii, número 5267, jueves 2 de mayo de 2019. Recuperado el 3 de mayo de 2019, de http:/ / gaceta.diputados.gob.mx/

Reporte Índigo (18 diciembre 2018). "Piden rectores de universidades estatales al congreso y a amlo rectificar presupuesto". Recuperado el 29 de abril de 2019, de https://www.reporteindigo.com/reporte/piden-rectores-de-universidades-estatales-al-congreso-y-a-amlo-rectificar-presupuesto/

Rodríguez Aceves, Nora (20 diciembre 2018), "Un errorcito, el recorte a las universidades: Urzúa Macías". Siempre. Recuperado el 10 de enero de 2019, de http:/ / www.siempre.mx/2018/11/recorte-presupuesto-educacion/

Román, José Antonio (17 diciembre 2018). “Rechaza uam recorte presupuestal; pide al Congreso modificaciones". La Jornada. Recuperado el 6 de enero de 2019, de file:///G:/A\%20AAA\%20ARTÍCULO\%20PEF\%202019\%20 MARZO\%2019/Rechaza\%20UAM\%20recorte\%20presupuestal;\%20pide\%20 al \%20Congreso \%20modificaciones \% 20-\%20La \% 20Jornada.html

SHCP (2017). Presupuesto de Egresos de la Federación. Ejercicio Fiscal 2018. Recuperado el 25 de febrero de 2019, de https:/ / www.pef.hacienda.gob.mx/es/PEF2018

SHCP (2018a). Proyecto de Presupuesto de Egresos de la Federación. Ejercicio Fiscal 2019. Exposición de motivos. Recuperado el 25 de febrero de 2019, de https://www.ppef.hacienda.gob.mx/work/models/PPEF2019/docs/exposicion/EM_Capitulo_1.pdf

SHCP (2018b). Proyecto de Presupuesto de Egresos de la Federación. Ejercicio Fiscal 2019. Recuperado el 25 de febrero de 2019, de https:/ / www.ppef.hacienda.gob.mx/es/PPEF2019

SHCP (2018c). Presupuesto de Egresos de la Federación. Ejercicio Fiscal 2019. Recuperado el 25 de febrero de 2019, de https://www.pef.hacienda.gob.mx/ es/PEF2019

SHCP (2019a). Comunicado uif No 02-2019. La uif anuncia acciones de prevención y detección de lavado de dinero en contra de una universidad pública estatal. Recuperado el 8 de mayo de 2019, de https://www.gob.mx/shcp/ prensa/comunicado-uif-no-02-2019-la-uif-anuncia-acciones-de-prevenciony-deteccion-de-lavado-de-dinero-en-contra-de-una-universidad-publicaestatal?idiom $=\mathrm{es}$

SHCP (2019b). Cuenta de la Hacienda Pública Federal. Recuperado el 7 de enero de 2019, de https://www.finanzaspublicas.hacienda.gob.mx/es/Finanzas_Publicas/Cuenta_Publica

Soberanes Díez, José María (2018). "La gratuidad de la educación pública en la jurisprudencia de la Suprema Corte de Justicia de la Nación". México. Revista Mexicana de Derecho Constitucional, Núm. 39, julio-diciembre 2018.

Tuirán, Rodolfo (2019). “Obligatoriedad y la gratuidad de la educación superior, ¿a qué costo?", en Nexos, 20 de marzo de 2019. Recuperada el 20 de mayo de 2019, de https:/ / educacion.nexos.com.mx/?p=1696

Ugalde, Luis Carlos (Coordinador) (2014). La negociación política del presupuesto en México 1997-2012. México, Integralia Consultores, Senado de la República, SITESA. 